\title{
Visualization of Hyaluronic Acid in the Anterior Segment of Rabbit and Monkey Eyes
}

\author{
ELKE LÜTJEN-DRECOLL**, MONA SCHENHOLM ${ }^{b}$, ERNST TAMM ${ }^{*}$ \\ AND ANDERS TENGBLAD ${ }^{c}$ \\ ${ }^{2}$ Department of Anatomy, University of Erlangen-Nürnberg, Erlangen, F.R.G., 'Department of \\ Experimental Ophthalmology and ${ }^{\circ}$ Biomedical Research, Pharmacia AB, Uppsala, Sweden
}

(Received 4 August 1989 and accepted in revised form 16 November 1989)

\begin{abstract}
Hyaluronic acid was visualized using a new histochemical technique : the hyaluronic acid-binding region of the cartilage proteoglycan was isolated, linked with biotin and used in histological sections as a ligand for hyaluronic acid. Staining was performed using the avidin-biotin-peroxidase technique.

The presence of hyaluronic acid in the anterior segments of rabbit and monkey eyes was studied in fresh-frozen, as well as in fixed paraffin sections; addition of cetyl-pyridinium chloride to the fixative was essential.

In both species intense staining was seen in the stroma of the conjunctiva, the connective tissue of the ciliary processes, and on the apical membranes of the corneal endothelium. Species differences were observed in both conventional and unconventional outflow pathways. In rabbits, the tissue located directly adjacent to the aqueous plexus and the connective tissue surrounding the poorly developed ciliary muscle were stained. In monkeys, only the anterior, non-filtering part of the trabecular meshwork and the outer wall of Schlemm's canal showed intense staining in paraffin sections. In frozen sections. the inner wall (cribriform region of the trabecular meshwork) was also stained. The spaces between the anterior part of the ciliary muscle bundles remained almost unstained.

Key words: hyaluronic acid; hyaluronan; histochemistry; cornea; trabecular meshwork; conjunctiva ; ciliary body; iris.
\end{abstract}

\section{Introduction}

Since Bárány found that resistance to aqueous outflow in non-primate eyes is greatly reduced following anterior chamber perfusion with hyaluronidase (Bárány, 1953; Bárány and Scotchbrook, 1954; Bárány and Woodin, 1955: Bárány, 1956), many investigators have tried to localize hyaluronic acid (HA) and other glycosaminoglycans (GAGs) in the anterior segment of the eyes of various species. Different methods were used for this purpose, i.e. the replica technique (Vrabec, 1957; Berggren and Vrabec, 1957; Wolf, 1968), or histochemical and ultrahistochemical methods using toluidine blue (Ashton, Brini and Smith, 1956), cresyl violet (Vrabec, 1957). colloidal iron (Zimmerman, 1957: Duke and Siegelman, 1961 : Armaly and Wang, 1975: Grierson and Iee, 1975), alcian-blue (Duke and Siegelman, 1961 ; Armaly and Wang, 1975 ; Lütjen-Drecoll. Futa and Rohen, 1981) or ruthenium red (Segawa, 1975; Harnisch, 1976: Grierson, Lee and Abraham, 1977; Mizokami, 1977: Richardson, 1982). All these methods, however, generally lack both sensitivity and specificity for differentiating between various GAGs, even if they are used in conjunction with enzymatic digestions of tissue sections.

* For reprint requests at: Anatomisches Institut, Lehrstuhl II der Universität Erlangen-Nürnberg, Krankenhausstraße 9. D-8520 Erlangen. F.R.G.
Recently an affinity purification method has been developed to isolate a protein from cartilage which specifically binds to HA. This probe, the HA-binding region (HABR) of the aggregating proteoglycans, has been used both to detect HA in tissues and body fluids, and also to demonstrate $\mathrm{HA}$ in tissue sections (Nettelbladt et al., in press). The HABR binds to HA with high specificity and affinity (Hardingham and Muir, 1972; Hascall and Heinegård, 1974; Hascall and Hascall, 1981).

In the present investigation it was used to study the distribution of HA in the anterior segment of the eyes of two species, the cynomolgus monkey (Macaca fascicularis), and the albino rabbit, respectively. HA was precipitated in the tissue with cetyl-pyridinium chloride $(\mathrm{CPC})$ in order to reduce loss of HA during preparation and staining.

\section{Materials and Methods}

Four adult albino rabbits (New Zealand White) and four cynomolgus monkeys (Macaca fascicularis), kept under standardized conditions and given food and tap water ad libitum were used. Before enucleation of the eyes, the animals were killed by an overdose of pentobarbital. The eyes were cut at the equator posterior to the ora serrata, and the anterior segments were either immersed in fixative or deep frozen in liquid nitrogen after immersion in isopentane. 


\section{Fixation}

To test various fixatives before embedding in paraffin, specimens were immersed in Bouin's fluid, methanol, Carnoy's fluid, $4 \%$ buffered formaldehyde containing $1 \% \mathrm{CPC}$, or in $2 \%$ glutaraldehyde (GA) dissolved in $0 \cdot 1 \mathrm{M}$ phosphate buffer, $\mathrm{pH} 7 \cdot 4$, with or without $1 \% \mathrm{CPC}$.

The best fixative was found to be $1 \%(\mathrm{v} / \mathrm{v}) \mathrm{CPC}$ in $4 \%(\mathrm{w} / \mathrm{v})$ buffered formaldehyde. After a fixation time of up to 1 month, no decrease in staining could be observed. The fixation was followed by dehydration of the tissues through graded ethanols, and consequently embedding in paraffin.

\section{Preparation of Biotinylated Probes for the Localization of Hyaluronate}

The HA-binding region (HABR) of the cartilage proteoglycan was isolated by affinity chromatography on $\mathrm{H} \Lambda$-sepharose from $4 \mathrm{~m}$ guanidine hydrochloride (GndHCL) extract of bovine nasal cartilage as described earlier (Tengblad, 1979). Biotin was linked to the purified HABR according to the principles outlined by Ripellino et al. (1985). In the present study, the linkproteins were excluded, since they are known to self aggregate, a fact that might add to unspecific interaction. Furthermore, the link-proteins may also bind to proteoglycans in the tissue. The HABR-HA complex, with a concentration of $1 \mathrm{mg}$ protein $\mathrm{ml}^{-1}$, was mixed, per ml, with $100 \mu \mathrm{l} N$-hydroxy succinimidobiotin $2 \mathrm{mg} \mathrm{ml}^{-1}$ (Sigma Co, Poole, U.K.). The solution was kept at room temperature for $30 \mathrm{~min}$ and then applied to a column of sephadex G15, total volume $7 \mathrm{ml}$ (PD-10, Pharmacia LKB Biotechnology $\mathrm{AB}$, Uppsala, Sweden) and eluted with $0.15 \mathrm{M}$ sodium chloride.

High molecular weight material recovered in the void volume was mixed with $\mathrm{GndHCl}$ to a concentration of $4 \mathrm{M}$, and $2.5 \mathrm{ml} \mathrm{HA}$-sepharose in $4 \mathrm{M} \mathrm{GndHCl}$; $0.5 \mathrm{M}$ sodium acetate $\mathrm{pH} 5.8$ was added per $\mathrm{mg}$ of protein. The gel slurry was dialyzed against 10 volumes of water for $20 \mathrm{hr}$ at room temperature. The gel was packed in a column, washed with 1,2 and $0.15 \mathrm{M}$ sodium chloride in $0.5 \mathrm{M}$ sodium acetate, $\mathrm{pH} 5.8$ ( $10 \cdot 7$, respectively, 2 gel columnes). In this way, the HA that had protected the binding site and any protein that had been destroyed during the labelling procedure were separated from the HABR-biotin complex.
The biotin-labelled hyaluronate binding region (HABR-biotin) was then recovered from the gel with $4 \mathrm{M} \mathrm{GndHCl}$ in $0.5 \mathrm{~m}$ sodium acetate, $\mathrm{pH} 5.8$, dialyzed against $0.15 \mathrm{~m}$ sodium chloride and concentrated on a UM 10 membrane (Amicon Division, W. R. Grace and Co., Danvers, MA). The HABR-biotin was stored at $-20^{\circ} \mathrm{C}$ at a concentration of $0.18 \mathrm{mg} \mathrm{ml}^{-1}$ until further use. A long time storage at $2-7^{\circ} \mathrm{C}$ with and without acetic acid (0.01\%) was found to give less stability (1-2 months). and at room temperature degradations appeared within days. At $-20^{\circ} \mathrm{C}$ the material was stable for more than 26 months.

\section{Histochemical Method}

HA was visualized by staining the probe using the avidine-biotin-complex ( $\mathrm{ABC}$ ) technique (Hsu, Raine and Fanger, 1981). The $A B C$ was conjugated with peroxidase.

\section{Paraffin Sections}

Five-micrometer paraffin sections were incubated (30 min) with bovine serum albumin $\left(10 \mathrm{mg} \mathrm{ml}^{-1}\right.$; Fraction V, A4503, Sigma, St Louis, MO) in phosphate buffered saline (PBS), and thereafter for $3 \mathrm{hr}$ at room temperature with $\mathrm{HABR}$-biotin $\left(20 \mu \mathrm{g} \mathrm{ml}^{-1}\right)$.

After rinsing ( $2 \times 5 \mathrm{~min}$ in PBS), the sections were incubated for $1 \mathrm{hr}$ with $\mathrm{ABC}$ Vectastain ${ }^{\text {win }}$ Reagent (Vector Laboratories Inc, Burlingham, CA) and rinsed in PBS $(2 \times 5 \mathrm{~min})$. The sections that were now labelled with peroxidase were visualized with a substrate solution prepared as follows: $10 \mathrm{mg} 3-$ amino-9-ethylcarbazole (Karl Roth, Karlsruhe, F.R.G.) in $6 \mathrm{mi}$ dimethylsulphoxide (Merck Darmstadt, F.R.G.).

This solution was added to $40 \mathrm{ml}$ acetate buffer, pH 5.0, containing $5 \mu \mathrm{l} \mathrm{H}_{2} \mathrm{O}_{2}$. The sections were incubated in this solution for $5 \mathrm{~min}$ and rinsed in tap water. Some sections were counterstained in Mayers Haematoxylin. The sections were mounted under glass coverslips in Kaisers glycerin-gelatin and examined by light microscopy.

Sections for negative control were incubated for $2 \mathrm{hr}$ in a humified chamber at $37^{\circ} \mathrm{C}$ with 50 units $\mathrm{ml}^{-1}$ of Streptomyces hyaluronidase (Seikagaku Fine Biochemicals, Tokyo, Japan) in $100 \mathrm{~mm}$ sodium acetate buffer $\mathrm{pH} 5.8$, in the presence of protease inhibitors, $1.8 \mathrm{~mm}$ ethylenediamine tetroacetic acid (EDTA, Merck Darmstadt, F.R.G.).

FIG. 1.a, Paraffin section through the human umbilical cord stained for HA $(\times 240)$. The mucous connective tissue (Wharton's jelly) stains positive (asterisks). b. Staining of the umbilical cord after preincubation with Streptomyces hyaluronidase $(\times 240)$. The mucous connective tissue remains unstained.

FIG. 2. Paraffin section through the anterior segment of a rabbit eye stained for HA $(\times 50)$. The connective tissue of the ciliary body $(\mathrm{CB})$ is intensely stained. Staining is also seen surrounding the collector channels (CC) in the sclera, in the tissue directly adjacent to the aqueous plexus (AP) (arrows), and on the apical surface of the corneal endothelium (open arrow). The staining extends towards the cells covering the iris strands of the pectinate ligament (arrowhead). A weak staining occurs in the iris stroma (I) and in the anterior part of the sclera (SC) in the vicinity of the collector channels. The posterior parts of the sclera remain unstained. $\mathrm{R}=$ remnants of vitreous. 

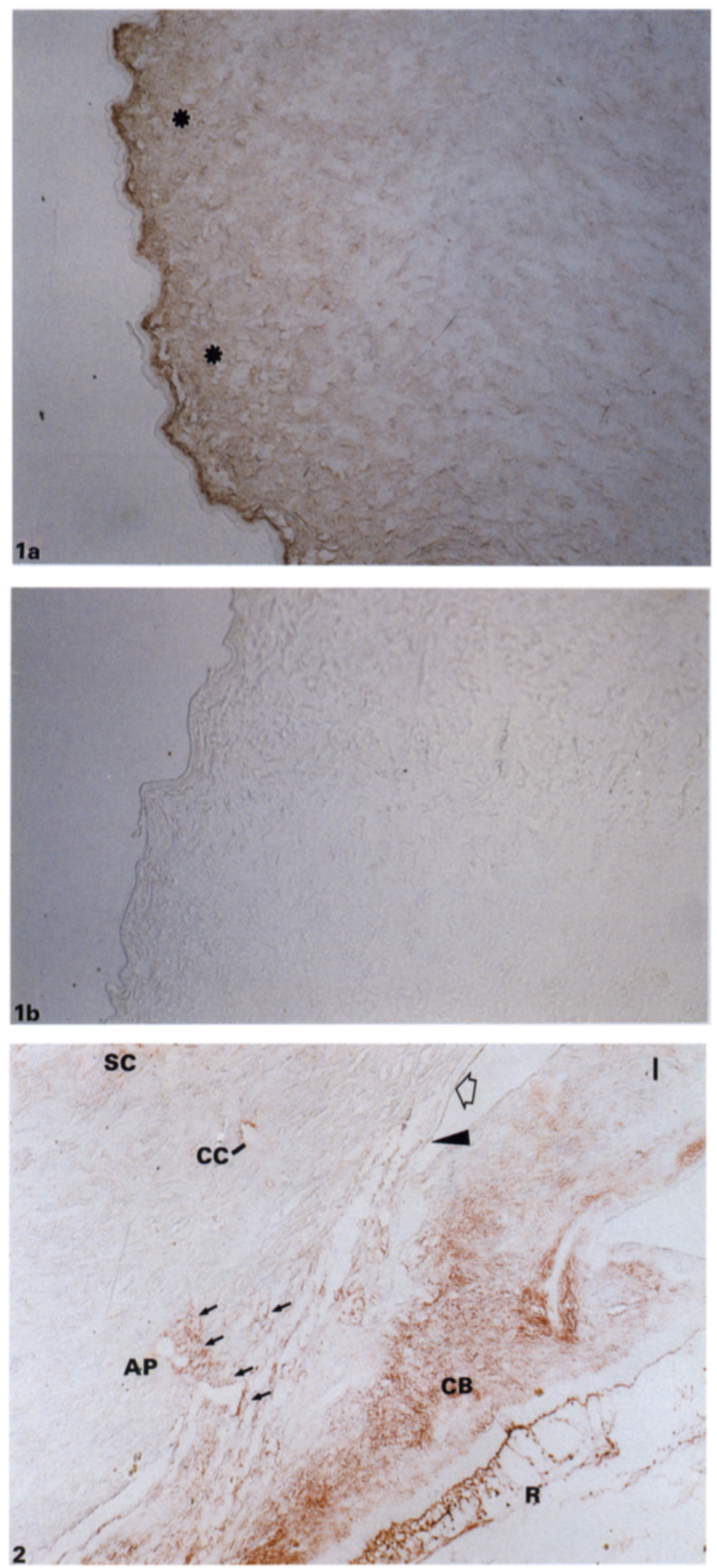

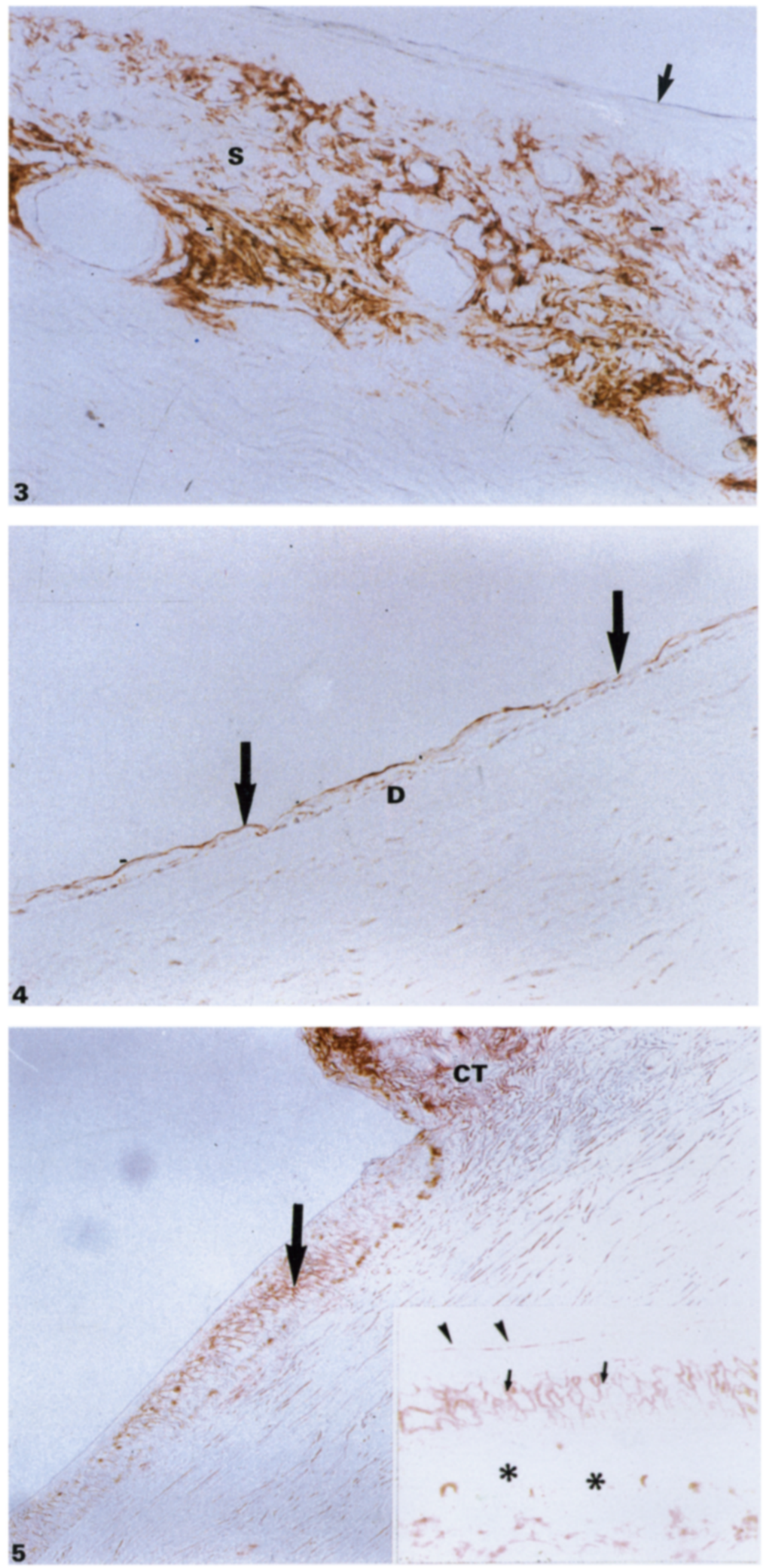
Soyabean trypsin inhibitor $\left(1.8 \mu \mathrm{g} \mathrm{ml}^{-1}\right.$, Worthington Freehold, NJ), $2.0 \mathrm{~mm}$ iodoacetic acid (Sigma), 0.18 mM 6-amino-n-caproicacid (Sigma). $9.90 \mathrm{~mm}$ bensamidine (Sigma) and $1.8 \mu \mathrm{g} \mathrm{ml}^{-1}$ pepstatin A (Sigma).

For positive control, fresh human umbilical cord obtained from a local hospital was used. The material was fixed in $1 \% \mathrm{CPC}$ in $4 \%$ buffered formalin. Staining was performed according to the protocol described above. For inhibition of endogenous peroxidase the sections were treated with $1 \% \mathrm{H}_{2} \mathrm{O}_{2}$ for 5 min before incubation with HABR-biotin.

\section{Fresh-frozen Tissues}

Sections $(5-10 \mu \mathrm{m})$ were cut in a cryostat at $20^{\circ} \mathrm{C}$. The incuhation time was $2 \mathrm{hr}$ both for HABR-biotin and control with Streptomyces hyaluronidase. The other procedures were the same as described for the paraffin sections.

\section{Results}

Staining of HA depends on the concentration and amount of HA within the tissue. Despite the fact that CPC was added to the fixative, there was a significant staining difference between frozen and paraffin sections. In nearly all areas investigated, staining was much more pronounced in the frozen sections, indicating loss of $\mathrm{HA}$ during the paraffin embedding procedures. Loss of HA was most pronounced in loose tissues. When CPC was omitted from the fixative solution, no staining was observed. CPC-formalin fixed human umbilical cord showed intense staining in the mucous connective tissue (Wharton's jelly) [Fig. 1(a)]. No staining was seen in the hyaluronidase-treated control sections [Fig. 1(b)].

\section{Rabbit}

Intense staining for $\mathrm{HA}$ was seen in the connective tissue of the ciliary body (Fig. 2) and in the connective tissue of the conjunctiva (Fig. 3). In rabbits, only few muscle cells form the ciliary muscle. Most of the ciliary body consists of connective tissue. This connective tissue surrounding the ciliary muscle fibers stains intensively in both frozen and paraffin sections. Staining in the connective tissue of the conjunctiva, which was also present in frozen as well as in paraffin sections, was seen in the limbal and fornix region, as far as the conjunctiva was included in the section.

Less intense staining, seen also in both frozen and paraffin sections, occurred on the apical cell membranes of the corneal endothelium (Fig. 4) and in the tissue directly adjacent to the aqueous plexus and the collector channels (Fig. 2). Staining of the corneal endothelium extended towards the cells covering the iris strands of the pectinate ligament (Fig. 2). Corneal stroma (Fig. 4) as well as conjunctival (Fig. 3) and corneal epithelium did not stain.

Weak staining occurred in the stroma of the iris (Fig. 2) and in the anterior portion of the sclera where the collector channels and aqueous veins pass through the sclera (Fig. 2). No staining was seen further posterior. In the iris staining was not distributed evenly within the whole stroma, but was mainly seen in the middle and posterior part of the stroma adjacent to the dilator muscle. The anterior surface facing the aqueous of the anterior chamber did not stain (Fig. 2).

\section{Monkey}

In both frozen and paraffin sections intense staining was only seen in the conjunctiva (Fig. 5). In contrast to the rabbits, staining was not only seen in the conjunctival stroma but also in the epithelium. Here. staining occurred around three to four cell layers in the middle of the epithelium and on the surface of the superficial cells (Fig. 5). The remaining cells showed little or no staining. This staining pattern was seen in the limbal conjunctiva as well as in the fornix region. Other parts of the conjunctiva were not investigated. Corneal epithelium and stroma did not stain.

Less intense staining was seen covering the apical cell membranes of the corneal endothelium (Fig. 6). This staining was also seen in both frozen and paraffin sections. The cells covering the inner surface of the operculum showed the same staining pattern and intensity as the corneal endothelium (Fig. 6).

A similar staining intensity occurred in the anterior non-filtering part of the trabecular meshwork and directly underneath the outer wall endothelium (Fig. 6). In the ciliary body, the connective tissue underlying the ciliary epithelium stained similarly (Fig. 7). In frozen sections, staining was also seen underneath the inner wall endothelium in the so-called cribriform region (Fig. 8).

In the iris. staining was seen in the anterior surface

FIG. 3. Paraffin section through the limbal conjunctiva of a rabbit eye stained for HA $(\times 240)$. The stroma (S) of the conjunctiva shows an intense staining while the conjunctival epithelium (arrow) remains unstained.

FIG. 4. Higher magnification of the corneal endothelium of a rabbit eye stained for HA $(\times 240)$. The apical surface of the endothelium shows an intense staining (arrows) while the basal surface facing Descemet's membrane (D) remains unstained.

FIG. 5. Paraffin section through the limbus region of a monkey eye stained for HA $(\times 120)$. The stroma of the conjunctiva (CT) is intensely stained. Staining is also seen surrounding the conjunctival epithelial cells (arrow). Inset: Higher magnification of the conjunctival cpithelium $(\times 240)$. Note that staining is seen surrounding the cells in the middle portion of the epithelium (arrows) and on top of the superficial cells (arrowheads). The cell membranes of the superficially located cells and of the cells adjacent to the basal lamina are not stained (asterisks). 

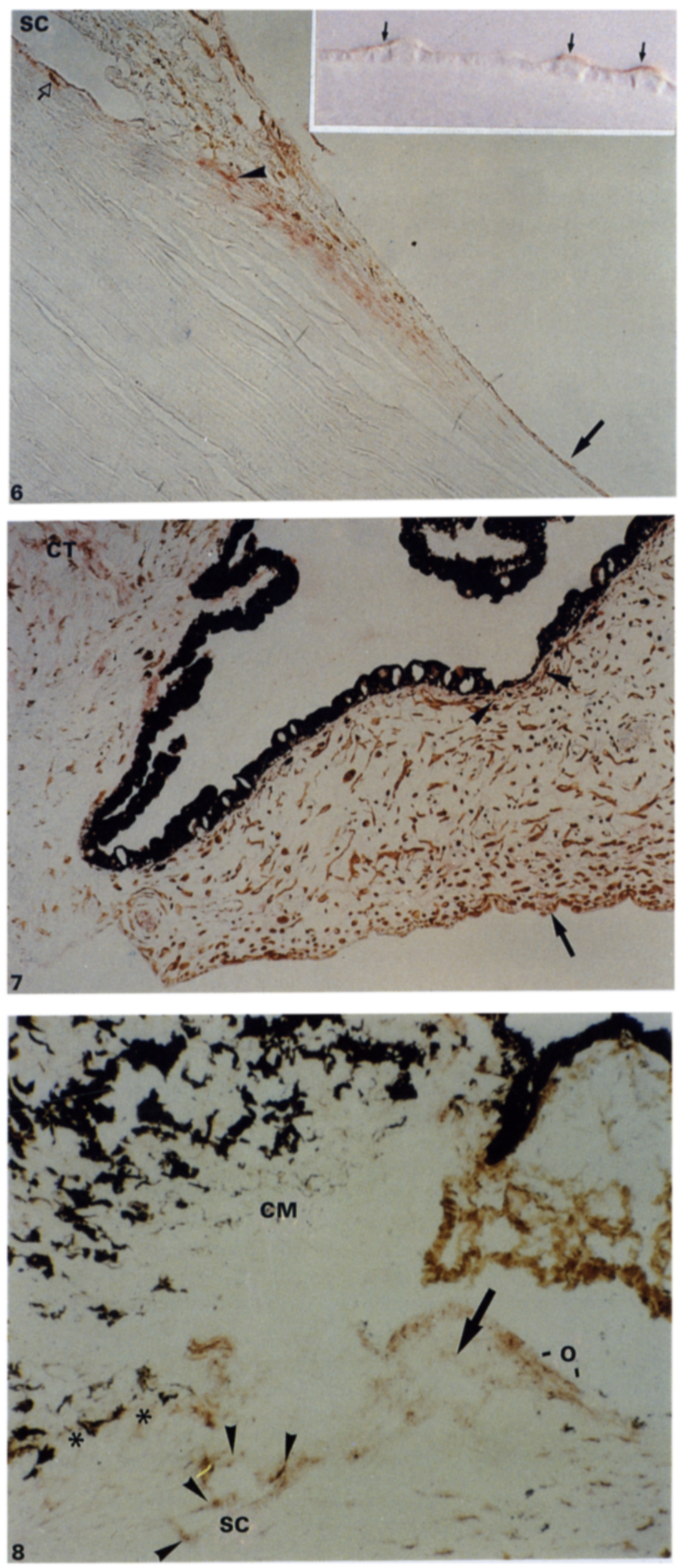
facing the anterior chamber only, while the stroma adjacent to the dilator muscle remained nearly unstained (Fig. 7).

Weak staining, which occurred in frozen sections only, was seen in the central core of the trabecular lamellae (Fig. 8). Weak staining was also seen in the suprachoroidal cleft (Fig. 8).

In the ciliary muscle, weak staining was present in the circular and reticular portion, as well as in the posterior longitudinal portion. Staining was seen directly surrounding the ciliary muscle bundles but not in the spaces between the bundles. No staining was observed in the anterior longitudinal portion (Fig. 8).

\section{Discussion}

The staining differences between frozen and paraffin sections indicate loss of HA from the tissues during fixation and embedding procedures. We do not know whether there is also loss of HA in frozen sections, and realize that lack of staining does not prove lack of $\mathrm{HA}$ in vivo.

On the other hand our histochemical results are comparable with those obtained by biochemical methods. For instance, in both species studied, we found staining for HA in the anterior part of the scleral stroma, but not in the corneal stroma.

HA was biochemically demonstrated in the sclera of human, bovine and rabbit eyes (Meyer et al., 1953: Polatnick, laTessa and Katzin, 1957; Breen et al., 1972: Borcherding et al., 1975; Knepper, Farbman and Telser, 1981), whereas it was not found in the adult corneal stroma (Breen et al., 1972; Borcherding et al., 1975). It has been suggested by these authors that the absence of HA may be important for the precisely ordercd spacing of collagen fibrils in the cornea.

In agreement with biochemical studies (Knepper et al.. 1981: Acott et al., 1985), there is also the fact that staining was found in the aqueous outflow pathways. Histochemically, the intensity of the staining was, however, different in the two species. Staining was more intense in rabbits than in monkeys. In monkey eyes only the non-filtering anterior part of the meshwork showed an intense staining, whereas the cribriform meshwork underneath Schlemm's canal, and the central core of the trabecular lamellae were only stained weakly. No staining was seen in the intertrabecular spaces. In contrast, in rabbit eyes the whole reticular meshwork stained intensely in all areas. If $\mathrm{HA}$ is at least partly responsible for the resistance to outflow in rabbit eyes, our finding could explain why infusion of hyaluronidase has a more marked effect on outflow facility in rabbit than in monkey eyes (Peterson and Jocson, 1974; Knepper, Fabman and Telser, 1984).

While staining of the conjunctival stroma was observed in both species, staining of the conjunctival epithelium was present in monkeys only. It is known that rabbit conjunctival epithelium shows structural differences to primate conjunctival epithelium (Steuhl and Rohen, 1984), which could be related to the specialized lid apparatus of lagomorphs. HA in monkey conjunctival epithelium might be responsible for the functional important water binding capacity. A similar staining for HA was reported in the spinous cell layer of human epidermis (Tammi et al., 1988). The authors discussed that HA might also facilitate cellular detachment in cell layers, where upward migration of cells takes place. The reasons why there are staining differences in monkey conjunctival epithelium are not known.

Interestingly, intense staining was seen in both species on the apical surfaces of the endothelial cells covering the operculum in monkeys, and the iridial strands of the pectinate ligament in rabbits. A similar staining was also seen on the apical surface of the corneal endothelium. Recent binding studies with tritiated HA showed that the corneal endothelium has specific binding sites for HA (Madsen et al., in press). A layer of 'mucous material' on the corneal endothelium in primate eyes has already been described by Wolf $(1968,1969)$ and by Sperling and Jacobsen (1980). Wolf suggested that this mucous layer is produced by the corneal endothelium and is then transported to the trabecular meshwork. Bárány, Berggren and Vrabec (1957) found a thick hyaluronidase-sensitive coating covering the corneal cndothclium in the Tawny owl. These authors discuss a self-sealing effect of this layer.

Differences in the staining pattern and intensity between rabbit and monkey eyes were also seen in the uvea. In the iris of monkey eyes the most intense

FIG. 6. Paraffin section through the peripheral cornea and the anterior part of the trabecular meshwork of a monkey eye stained for HA $(\times 120)$. Staining is seen on the apical membrane of the corneal endothelium (arrow) and in the non-filtering anterior part of the trabecular meshwork (arrowhead). No staining is seen in the filtering part of the meshwork, while the outer wall of Schlemm's canal (SC) shows positive staining (open arrow). Inset: Higher magnification of the corneal endothelium $(\times 240)$. Adjacent to the apical cell membrane intense HA staining is seen (arrows).

Fig. 7. Paraffin section through the iris and anterior part of the ciliary body of a monkey eye stained for HA $(\times 120)$. The ciliary body staining is seen in the connective tissue of the processes (CT). In the iris, the anterior surface (arrow) and the region of the dilator muscle (arrowheads) are stained.

FIG. 8. Frozen section through the chamber angle region of a monkey eye stained for HA $(\times 120)$. In frozen sections, staining of the opercular region $(0)$, the anterior non-filtering part of the meshwork (arrow), the inner and outer wall and the so-called cribriform region (arrowheads) of Schlemm's canal (SC) is more intense than in paraffin sections. Staining is also seen in the suprachoroidal cleft (asterisks) between ciliary muscle (CM) and sclera. 
staining was seen at the anterior surface of the iris. whereas in rabbits this region remained unstained. In contrast to the rabbit, the anterior surface of the iris in monkeys is not covered by a continuous epithelium. and with conventional staining methods, the stroma appears to be open to the aqueous through crypts and fissures (Rohen and Voth, 1960). But HA could build a kind of barrier between aqueous and iris stroma and vessels. Evidence for an iris delay in the diffusion of [ $\left.{ }^{125} \mathrm{I}\right]$ - and $\left[{ }^{131} \mathrm{I}\right] \boldsymbol{o}$-iodohippurates between plasma and aqueous humour was found in cynomolgus monkeys (Wilson and Bárány. 1983). The morphological investigation of the irides of these animals showed that those eyes with a very low entry coefficient of the substances from the iris vessels into the anterior chamber showed particularly large numbers of optically empty spaces in the anterior boundary layer of the iris (Ernst. 1987). Our findings indicate that these optically empty spaces are filled with HA. We do not know how the HA influences the diffusion of the iodohippurate.

The ciliary body of the monkey eyes shows only little HA staining between the muscle bundles, whereas in the rabbit, the connective tissue surrounding the ciliary muscle as well as the connective tissue anterior to the muscle appears intensely stained. If $\mathrm{HA}$ in the ciliary body also causes resistance to aqueous outflow, the staining difference could explain why uveoscleral outflow is much less in rabbits than in monkeys (Bill. 1966a, b).

Even though our new staining method helps to understand the physiological differences between monkey and rabbit eyes better, a direct correlation between the amount of staining and physiological data is still not possible. A better method to fix HA in loose connective tissues is needed to obtain histochemical results in embedded specimens as well, which would give a better morphology, especially in the outflow pathways.

\section{References}

Acott. T. S.. Westcott, M., Passo, M. S. and van Buskirk, E. M. (1985). Trabecular meshwork glycosaminoglycans in human and cynomolgus monkey eyes. Invest. Ophthalmol. Vis. Sci. 26, 1320-9.

Armaly, M. F. and Wang. Y. (1975). Demonstration of acid mucopolysaccharides in the trabecular meshwork of the rhesus monkey. Invest. Ophthalmol. Vis. Sci. 14 , 507-16.

Ashton, N., Brini, A. and Smith, R. (1956). Anatomical studies of the trabecular meshwork of the normal human eye. Br. J. Ophthalmol. 40, 257-82.

Bárány, E. H. (1953). In vitro studies of the resistance to flow through the angle of the anterior chamber. Acta Soc. Med. Upsaliensis 59, 260-76.

Bárány, E. H. (1956). The action of different kinds of hyaluronidase on the resistance to flow through the angle of the anterior chamber. Acta Ophthalmol. 34. $397-403$.

Bárány, F. H., Berggren, L. and Vrabec. F. (1957). The mucinous layer covering the corneal endothelium in the owl strix aluco. Br. J. Ophthalmol. 41, 25-30.

Bárány, E. H. and Scotchbrook, S. (1954). Influence of testicular hyaluronidase on the resistance to flow through the angle of the anterior chamber. Actu Physiol. Scand. 30, 240-8.

Bárány, E. H. and Woodin, A. M. (1955). Hyaluronic acid and hyaluronidase in the aqueous humor and the angle of the anterior chamber. Acta Physiol. Siand. 33. $257-88$.

Berggren, L. and Vrabec, F. (1957). Demonstration of a coating substance in the trabecular meshwork of the eye and its decrease after perfusion experiments with different kinds of hyaluronidases. Am. J. Ophthalmol. 44. $200-8$.

Bill, A. (1966a). The routes for bulk drainage of aqueous humor in rabbits with and without cyclodialysis. Doc. Ophthalmol. 20, 157-69.

Bill, A. (1966b). Conventional and uveo-scleral drainage of aqueous humor in cynomolgus monkey (Macaca irus) at normal and high intraocular pressures. Exp. Eye Res. 5. 45-54.

Borcherding, M. S., Blacik, L. J., Sittig, R. A., Bizzell, J. W., Breen. M. and Weinstein, H. G. (1975). Proteoglycans and collagen fibre organization in human corneoscleral tissue. Exp. Eye Res. 21. 59-70.

Breen, M., Johnson, R. L.. Sittig, R. A., Weinstein, H. G. and Veis, A. (1972). The acidic glycosaminoglycans in human fetal development and adult life: Cornea, sclera and skin. Connect Tissue Res. 1, 291-303.

Duke, J.R. and Siegelmann. S. (1961). Acid mucopolysaccharides in the trabecular meshwork of the chamber angle. Arch. Ophthalmol. 66. 399-404.

Ernst. H. (1987). Quantitative licht- und elektronenmikroskopische Untersuchungen der Irisvorderfläche. Eine funktionell anatomische Studie bei Macaca fascicularis. Inaugural-Dissertation der Medizinischen Fakultät der Friedrich-Alexander-Universität: Erlangen-Nürnberg. F.R.G.

Grierson, I. and Lee, W. R. (1975). Acid mucopolysaccharides in the outflow apparatus. Exp. Fye Res. 21. $417-31$.

Grierson, 1., Lee, W. R. and Abraham. S. (1977). The appearance of the outflow apparatus of the eye after staining with ruthenium red. Acta Ophthalmol. 55. $827-36$.

Hardingham. T. E. and Muir, H. (1972). The specific interaction of hyaluronic acid with cartilage proteoglycans. Biochem. Biophys. Acta 279, 401-5.

Harnisch, J.-P. (1976). Elektronenmikroskopische darstellung saurer mukopolysaccharide im trabekelwerk. Klin. Mbl. Augenheilk. 169, 90-4.

Hascall, V. C. and Heinegård, D. (1974). Aggregation of cartilage proteoglycans. J. Biol. Chem. 249, 4232-56.

Hascall, V. C. and Hascall. G. K. (1981). Proteoglycans. In Cell Biology of the Extracellular Matrix (Ed. Hay, E. D.). Pp. 39-63. Plenum Press. New York.

Hsu. S. M., Raine. I. and Fanger, H. (1981). Use of avidin-biotin-peroxidase complex $(\mathrm{ABC})$ in immunoperoxidase techniques: $A$ comparison between $A B C$ and unlabeled antibody (PAP procedures). J. Histochem. Cytochem. 29,577-80.

Knepper, P. A.. Farbman. A. I. and Telser, A. G. (1981). Aqueous outflow pathway glycosaminoglycans. Exp. Eye Res. 32, 265-77.

Knepper, P. A., Farbman, A. I. and Telser. A. G. (1984). Fxogenous hyaluronidases and degradation of hyaluronic acid in the rabbit eye. Invest. Ophthalmol. Vis. Sci. 25. 286-93.

Lütjen-Drecoll. E.. Futa. R. and Rohen, I. W. (1981). 
Uitrahistochemical studies on tangential sections of the trabecular meshwork in normal and glaucomatous eyes. Invest. Ophthalmol. Vis. Sci. 21, 563-73.

Madsen, K., Schenholm, K., Jahnke, G. and Tengblad, A. Hyaluronate binding to intact corneas and cultured endothelial cells. Invest. Ophthalmol. Vis. Sci. (in press).

Meyer, K., Linker, A.. Davidson. E. A. and Weissmann, B. (19531. The mucopolysaccharides of bovine cornea. J. Biol. Chem. 205, 6-11.

Mizokami, K. (1977). Demonstration of masked acidic glycosaminoglycans in the normal human trabecular meshwork. Jpn. J. Ophthalmol. 21. 57-71.

Nettelblad, O., Bergh, J., Schenholm, M.. Tengblad, A. and Hällgren, R. Accumulation of hyaluronic acid in the alveolar interstitial tissue in bleomycin-induced alveolitis - a mechanism behind alveolar interstitial odema: Am. Rev. Resp. Dis, (in press).

Peterson, W. S. and Jocson, V. L. (1974). Hyaluronidase effects on aqueous outflow resistance. Am. J. Ophthalmol. $77,573-7$.

Polatnick, J., La Tessa, A. J. and Katzin, H. M. (1957). Comparison of bovine corneal and scleral mucopolysaccharides. Biochim. Biophys. Acta 26, 361-5.

Richardson. T. M. (1982). Distribution of glycosaminoglycans in the aqucous outflow system of the cat. Invest. Ophthalmol. Vis. Sci. 22, 319-29.

Ripellino. J. A.. Klinger, M. M., Margolis, R. U. and Margolis, R. K. (1985). The hyaluronic acid binding region as a specific probe for the localization of hyaluronic acid in tissue sections. J. Histochem. Cytochem. 33, 1060-6.

Rohen, J. W. and Voth, D. (1960). Zur Iris-Struktur der Primaten. Ophthalmologica 140, 27-33.

Segawa, K. (1975). Ultrastructural changes of the trabecular tissue in primary open angle glaucoma. Ipn $\nearrow$. Ophthalmol. 19. 317-38.

Sperling, S. and Jacobsen, S. R. (1980). The surface coat on human corneal endothelium. Acta Ophthalmol. 58, 96-101.

Steuhl, P. and Rohen. J. W. (1984). Cellular structure of the conjunctival epithelium of rabbits. Albrecht von Graefe's Arch. Klin. Exp. Ophthalmol. 221, 265-71.

Tammi, R., Ripellino, J. A., Margolis, R. U. and Tammi, M. (1988). Localization of epidermal hyaluronic acid using the hyaluronate binding region of cartilage as specific probe. J. Invest. Dermatol. 90, 412-4.

Tengblad, A. (1979), Affinity chromatography on immobilized hyaluronate and its application to the isolation of hyaluronate binding proteins from cartilage. Biochem. Biophys. Acta 578, 281-9.

Vrabec, F. (1957). The amorphous substance in the trabecular meshwork. Br. J. Ophthalmol. 41, 20-4.

Wilson, W.S. and Bárány, E. H. (1983). Iris delay, a neglected factor in aqueous humor dynamics. A study in the cynomolgus monkey (Macaca fascicularis). Exp. Eye Res. 37, 293-301.

Wolf. J. (1968). Inner surface of regions in the anterior chamber taking part in the regulation of the interocular tension, including the demonstration of the covering viscous substance. Docum. Ophthalmol. 25, 113-49.

Wolf, J. (1969). Transport of viscous coating from the region of the inner corneal surface into the fissures of the trabecular meshwork. Docum. Ophthalmol. 25, 227-47.

Zimmerman, L. E. (1957). Demonstration of hyaluronidase sensitive acid mucopolysaccharide in trabeculae and iris in routine paraffin sections of adult human eyes. Am. J. Ophthalmol. 44, 1-4. 